\title{
空间 $l^{p}(\Gamma)(p>1)$ 的单位球面间 等距算子的延拓 ${ }^{*}$
}

\author{
定光桂 $^{* *}$ \\ (南开大学数学系, 天津 300071)
}

\begin{abstract}
摘要 首先得到 $l^{p}(\Gamma)(p>1, p \neq 2)$ 单位球面之间 (满) 等距算子的表现定理, 然后
\end{abstract} 利用作者过去一个结果导出: 上述算子均可延拓为全空间上的 (实) 线性等距算子.

\section{关键词等距映像 严格凸}

Tingley ${ }^{[1]}$ 曾提出如下问题: 令 $E$ 和 $F$ 为实赋范空间, 且其单位球面为 $S_{1}(E)$ 和 $S_{1}(F)$. 设 $V_{0}: S_{1}(E) \rightarrow S_{1}(F)$ 是一个满等距 (即对于任意的 $x_{1}, x_{2} \in S_{1}(E)$, 都有 $\left\|V_{0}\left(x_{1}\right)-V_{0}\left(x_{2}\right)\right\|=$ $\left.\left\|x_{1}-x_{2}\right\|\right)$, 那么 $V_{0}$ 是否一定为一个 $E$ 上的线性或者仿射等距算子在 $S_{1}(E)$ 上的限制? 对于 复空间的情形, 答案显然是否定的. 举例来说: 令 $E=F=\mathbb{C}$ (复平面) 且 $V_{0}(x)=\bar{x}$, 即知.

作者 ${ }^{1)}$ 曾讲述过两单位球面间等距算子的延拓问题的来源和研究结果及状况, 并给出对 于 Banach 空间 $E$ 到连续函数空间 $C(\Omega)$ 中算子上述问题的一些结果. 而文献 [2] 则导出：当 $V_{0}$ 为 Hilbert 空间 $E$ 的单位球面 $S_{1}(E)$ 到 Hilbert 空间 $F$ 的单位球面 $S_{1}(F)$ “内” 的 1-Lipschitz 算子，当其值域满足条件 $-V_{0}\left[S_{1}(E)\right] \subset V_{0}\left[S_{1}(E)\right]$ 时, $V_{0}$ 必可延拓为 $E$ 到 $F$ 内的一个实线性 等距算子. 特别地, 可得到如下结果: 两个 Hilbert 空间的单位球面上定义的等距算子均可延拓 为全空间上的实线性等距算子. 本文将指出, 对于空间 $l^{p}(\Gamma)(p>1, p \neq 2, \Gamma$ 为任意指标集), 其 单位球面间满的等距算子亦均可延拓为全空间上的实线性等距算子. 注意到 Kakutani 的表现 定理 (文献 [3] 定理 2.7.1): “任何一个 $A L^{p}$ 空间 $(1 \leqslant p<\infty)$ 必序同构且等距同构于 $L^{p}(\Omega, \mu)$, 这里 $\Omega$ 为 Hausdorff 空间, $\mu$ 为 $\Omega$ 上 Baire 测度”, 对于由 “原子成长” 的 $A L^{p}$ 空间，我们导 出其单位球面上满的等距算子亦可延拓为全空间上的实线性等距算子.

下面讲述所得结果. 首先, 给出几个引理:

引理 $\mathbf{1}^{[2]}$ 设 $E, F$ 均为赋范空间且 $E$ 是严格凸的, $V_{0}$ 为单位球面 $S_{1}(E)$ 到 $S_{1}(F)$ 内的 等距算子. 如果有 $-V_{0}\left[S_{1}(E)\right] \subset V_{0}\left[S_{1}(E)\right]$ 和

$$
\left\|V_{0}\left(x_{1}\right)-V_{0}\left(x_{2}\right)\right\| \leqslant\left\|x_{1}-x_{2}\right\|, \quad \forall x_{1}, x_{2} \in S_{1}(E),
$$

那么 $V_{0}$ 必为 1-1 对应的, 且有 $V_{0}(-x)=-V_{0}(x), \forall x \in S_{1}(E)$.

2002-07-11 收稿

* 国家自然科学基金 (批准号 : 19971046) 和高校博士点基金资助项目

** E-mail: ding_gg@nankai.edu.cn

1) Ding G G. On the extension of isometries between unit spheres of $E$ and $C(\Omega)$. Acta Math Sinica (New Series) (待发表) 
引理 2 假设 $x, y \in l^{p}(\Gamma)$, 则有

$$
(\operatorname{supp} x) \cap(\operatorname{supp} y)=\varnothing \Longleftrightarrow\|x+y\|^{p}+\|x-y\|^{p}=2\left(\|x\|^{p}+\|y\|^{p}\right),
$$

这里, $\operatorname{supp} x=\{\gamma \mid x(\gamma) \neq 0, \gamma \in \Gamma\}, p \geqslant 1, p \neq 2$.

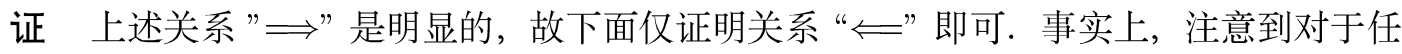

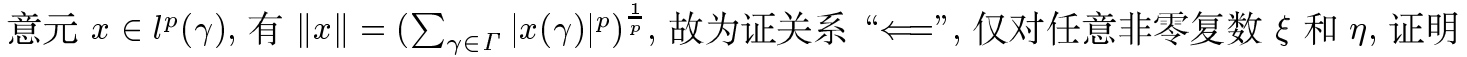

$$
|\xi+\eta|^{p}+|\xi-\eta|^{p} \neq 2\left(|\xi|^{p}+|\eta|^{p}\right)
$$

即可. 此可由著名的复数不等式 $(\forall \xi \neq 0, \eta \neq 0)$

$$
|\xi+\eta|^{p}+|\xi-\eta|^{p}<2\left(|\xi|^{p}+|\eta|^{p}\right) \quad(1 \leqslant p<2)
$$

和

$$
|\xi+\eta|^{p}+|\xi-\eta|^{p}>2\left(|\xi|^{p}+|\eta|^{p}\right) \quad(p>2)
$$

导出.

注 上述著名不等式有许多证明方法. 例如, 以上述第 2 关系式为例 $(p>2$ 情形), 先从 实函数 $t^{\frac{p}{2}}$ 的凸性得到

$$
\left(\frac{|\xi+\eta|^{2}+|\xi-\eta|^{2}}{2}\right)^{\frac{p}{2}} \leqslant \frac{1}{2}\left(|\xi+\eta|^{p}+|\xi-\eta|^{p}\right),
$$

再由复数的 “平行四边形法则” 及 $\frac{p}{2}>1$, 又可得到

$$
\left(\frac{|\xi+\eta|^{2}+|\xi-\eta|^{2}}{2}\right)^{\frac{p}{2}}=\left(|\xi|^{2}+|\eta|^{2}\right)^{\frac{p}{2}}>|\xi|^{p}+|\eta|^{p}, \quad \forall \xi \neq 0, \eta \neq 0
$$

从而导出结论.

引理 3 设 $V_{0}$ 为单位球面 $S_{1}\left[l^{p}(\Gamma)\right]$ 到 $S_{1}\left[l^{p}(\Delta)\right]$ 上的满等距算子 (这里 $\Gamma, \Delta$ 均为指标 集), $p>1, p \neq 2$, 那么

$$
(\operatorname{supp} x) \cap(\operatorname{supp} y)=\varnothing \Longleftrightarrow\left(\operatorname{supp} V_{0}(x)\right) \cap\left(\operatorname{supp} V_{0}(y)\right)=\varnothing .
$$

证 从 $V_{0}$ 的假设, 有

$$
\left\|V_{0}(x)-V_{0}(y)\right\|=\|x-y\|, \quad \forall x, y \in S_{1}\left[l^{p}(\Gamma)\right] .
$$

又由 $l^{p}(\Gamma)$ 当 $p>1$ 时为严格凸空间, 故从引理 1 可知

$$
V_{0}(-x)=-V_{0}(x), \quad \forall x \in S_{1}\left[l^{p}(\Gamma)\right],
$$

因此有

$$
\left\|V_{0}(x)+V_{0}(y)\right\|=\left\|V_{0}(x)-V_{0}(-y)\right\|=\|x+y\|, \quad \forall x, y \in S_{1}\left[l^{p}(\Gamma)\right],
$$

从而直接由引理 2 可以导出本结论.

引理 $4 V_{0}$ 如引理 3 所设, 那么

$$
\bigcup_{\gamma \in \Gamma} \operatorname{supp} V_{0}\left(e_{\gamma}\right)=\Delta
$$

这里 $e_{\gamma}=\left\{\xi_{\gamma^{\prime}} \mid \xi_{\gamma}=1, \xi_{\gamma^{\prime}}=0, \gamma^{\prime} \neq \gamma, \gamma^{\prime} \in \Gamma\right\}, \forall \gamma \in \Gamma$.

证 反之, 如果 $\bigcup_{\gamma \in \Gamma} \operatorname{supp} V_{0}\left(e_{\gamma}\right)=\Delta_{0} \neq \Delta$, 取一元 $y_{0} \in S_{1}\left[l^{p}(\Delta)\right]$, 使得 supp $y_{o} \subset \Delta \backslash \Delta_{0}$, 则有

$$
\left(\operatorname{supp} y_{0}\right) \cap\left(\operatorname{supp} V_{0}\left(e_{\gamma}\right)\right)=\varnothing, \quad \forall \gamma \in \Gamma
$$


由 $V_{0}$ 为满算子, 故必存在元 $x_{0} \in S_{1}\left[l^{p}(\Gamma)\right]$, 满足 $y_{0}=V_{0}\left(x_{0}\right)$. 由此, (2) 式即为

$$
\left[\operatorname{supp} V_{0}\left(x_{0}\right)\right] \cap\left[\operatorname{supp} V_{0}\left(e_{\gamma}\right)\right]=\varnothing, \quad \forall \gamma \in \Gamma,
$$

因而从引理 3 导出

$$
\left(\operatorname{supp} x_{0}\right) \cap\left(\operatorname{supp} e_{\gamma}\right)=\varnothing, \quad \forall \gamma \in \Gamma,
$$

也即 $x_{0}=\theta$, 与 $x_{0}$ 取法 $\left(\left\|x_{0}\right\|=1\right)$ 矛盾.

引理 5 在实空间情形, $V_{0}$ 为如上引理所设等距算子，则必存在由 $\Gamma$ 到 $\Delta$ 上的一个

“1-1” 对应满映像 $\sigma$, 以及一个绝对值为 1 的实数集 $\left\{\theta_{\gamma}\right\}$, 使得

$$
V_{0}\left(e_{\gamma}\right)=\theta_{\gamma} d_{\sigma(\gamma)},
$$

这里, $d_{\delta}=\left\{\eta_{\delta^{\prime}} \mid \eta_{\delta}=1, \eta_{\delta^{\prime}}=0, \delta^{\prime} \neq \delta, \delta^{\prime} \in \Delta\right\}, \forall \delta \in \Delta$.

证 首先证明, 对每一个 $\gamma \in \Gamma$, 均仅有一个 $\delta(\gamma) \in \Delta$, 使得 $\operatorname{supp} V_{0}\left(e_{\gamma}\right)=\delta(\gamma)$. 事实上, 由 $V_{0}\left(e_{\gamma}\right) \in S_{1}\left[l^{p}(\Delta)\right]$, 知 $\operatorname{supp} V_{0}\left(e_{\gamma}\right) \neq \varnothing$. 现设有某 $\delta \in \Delta$, 使得 $\delta \in \operatorname{supp} V_{0}\left(e_{\gamma}\right)$, 则有

$$
V_{0}\left(e_{\gamma}\right)=\left.V_{0}\left(e_{\gamma}\right)\right|_{\{\delta\}}+\left.V_{0}\left(e_{\gamma}\right)\right|_{\{\Delta \backslash \delta\}} .
$$

令

$$
y=\frac{\left.V_{0}\left(e_{\gamma}\right)\right|_{\{\delta\}}}{\left\|\left.V_{0}\left(e_{\gamma}\right)\right|_{\{\delta\}}\right\|},
$$

显然 $y \in S_{1}\left[l^{p}(\Delta)\right]$. 从 $V_{0}$ 为满算子, 故存在元 $x \in S_{1}\left[l^{p}(\Gamma)\right]$ 满足 $V_{0}(x)=y$, 从而由引理 3 导出

$$
\begin{aligned}
& {\left[\operatorname{supp} V_{0}(x)\right] \cap\left[\operatorname{supp} V_{0}\left(e_{\gamma^{\prime}}\right)\right] }=\left[(\operatorname{supp} y) \cap \operatorname{supp} V_{0}\left(e_{\gamma^{\prime}}\right)\right] \\
& \subset\left[\operatorname{supp} V_{0}\left(e_{\gamma}\right)\right] \cap\left[\operatorname{supp} V_{0}\left(e_{\gamma^{\prime}}\right)\right]=\varnothing, \\
& \forall \gamma^{\prime} \neq \gamma, \quad \gamma^{\prime} \in \Gamma .
\end{aligned}
$$

再次应用引理 3 , 则有

$$
(\operatorname{supp} x) \cap\left(\operatorname{supp} e_{\gamma^{\prime}}\right)=\varnothing, \quad \forall \gamma^{\prime} \neq \gamma, \quad \gamma^{\prime} \in \Gamma,
$$

故由 $x$ 取法, 由上述知存在 $\theta=1$ 或 -1 , 有 $x=\theta e_{\gamma}$. 利用引理 1 , 有

$$
y=\frac{\left.V_{0}\left(e_{\gamma}\right)\right|_{\{\delta\}}}{\left\|\left.V_{0}\left(e_{\gamma}\right)\right|_{\{\delta\}}\right\|}=V_{0}(x)=V_{0}\left(e_{\gamma}\right) \text { 或 }-V_{0}\left(e_{\gamma}\right),
$$

也即导出 $\operatorname{supp} V_{0}(x)=\delta$. 由此知对每个 $\gamma \in \Gamma, \delta(\gamma)=\delta$ 是惟一确定的. 然后作 $\Gamma$ 到 $\Delta$ 的映 像 $\sigma$ 为 $\sigma(\gamma)=\delta(\gamma)$. 注意到引理 4, 可知 $\sigma$ 为 “满” 映射. 下面证明 $\sigma$ 为 “ 1-1” 对应的. 事 实上, 反之如有 $\gamma_{1} \neq \gamma_{2}$, 但 $\sigma\left(\gamma_{1}\right)=\sigma\left(\gamma_{2}\right)=\delta$, 那么, 由 $V_{0}$ 假设及引理 1 , 有

$$
\left\|V_{0}\left(e_{\gamma_{1}}\right) \pm V_{0}\left(e_{\gamma_{2}}\right)\right\|=\left\|e_{\gamma_{1}} \pm e_{\gamma_{2}}\right\| \text {. }
$$

另一方面，从上段结果有

$$
V_{0}\left(e_{\gamma_{1}}\right)=\theta_{1} d_{\delta}, \quad V_{0}\left(e_{\gamma_{2}}\right)=\theta_{2} d_{\delta}, \quad\left|\theta_{1}\right|=\left|\theta_{2}\right|=1,
$$

因而又可导出

$$
\max \left\{\left\|V_{0}\left(e_{\gamma_{1}}\right)+V_{0}\left(e_{\gamma_{2}}\right)\right\|, \quad\left\|V_{0}\left(e_{\gamma_{1}}\right)-V_{0}\left(e_{\gamma_{2}}\right)\right\|\right\}=2 .
$$

此显然与上式矛盾, 因此本引理得证.

下面给出在实空间中单位球面 $S_{1}\left[l^{p}(\Gamma)\right]$ 到 $S_{1}\left[l^{p}(\Delta)\right]$ 上满等距算子的表现定理. 此显然为 Banach 的书 ${ }^{[4]}$ 中第 11 章第 5 节之相应定理的推广. 由于本文的等距算子仅仅是定义在单位 
球面上 (而原定理涉及的却是定义在全空间，并且还是线性的等距算子), 因此导出就不那么容 易了。

定理 1 在实空间中, 设 $V_{0}$ 为单位球面 $S_{1}\left[l^{p}(\Gamma)\right]$ 到 $S_{1}\left[l^{p}(\Delta)\right]$ 上的满等距算子 $(p>1, p \neq$ $2)$, 那么, 必存在一个从 $\Delta$ 到 $\Gamma$ 上的 “ 1-1” 对应满映像 $\pi$, 以及一族绝对值为 1 的实数集 $\left\{\theta_{\delta}\right\}$, 使得 $V_{0}(x)=\sum_{\delta \in \Delta} \theta_{\delta} \xi_{\pi(\delta)} d_{\delta}, \forall x=\sum_{\gamma \in \Gamma} \xi_{\gamma} e_{\gamma} \in S_{1}\left[l^{p}(\Gamma)\right]$.

证 对于任意元 $x=\sum_{\gamma \in \Gamma} \xi_{\gamma} e_{\gamma} \in S_{1}\left[l^{p}(\Gamma)\right]$, 设

$$
V_{0}(x)=\sum_{\delta \in \Delta} \xi_{\delta}^{\prime} d_{\delta}
$$

由引理 5, 对任意 $\xi_{\gamma_{1}} \neq 0$, 有

$$
\begin{aligned}
\left\|V_{0}(x)+\frac{\xi_{\gamma_{1}}}{\left|\xi_{\gamma_{1}}\right|} V_{0}\left(e_{\gamma_{1}}\right)\right\|^{p} & =\left\|\sum_{\delta \in \Delta} \xi_{\delta}^{\prime} d_{\delta}+\frac{\xi_{\gamma_{1}}}{\left|\xi_{\gamma_{1}}\right|} \theta_{\gamma_{1}} d_{\sigma\left(\gamma_{1}\right)}\right\|^{p} \\
& =\left\|\sum_{\gamma \in \Gamma} \xi_{\sigma(\gamma)}^{\prime} d_{\sigma(\gamma)}+\frac{\xi_{\gamma_{1}}}{\left|\xi_{\gamma_{1}}\right|} \theta_{\gamma_{1}} d_{\sigma\left(\gamma_{1}\right)}\right\|^{p} \\
& =\left\|\sum_{\gamma \neq \gamma_{1}} \xi_{\sigma(\gamma)}^{\prime} d_{\sigma(\gamma)}+\left(\xi_{\sigma\left(\gamma_{1}\right)}^{\prime}+\frac{\xi_{\gamma_{1}}}{\left|\xi_{\gamma_{1}}\right|} \theta_{\gamma_{1}}\right) d_{\sigma\left(\gamma_{1}\right)}\right\|^{p} \\
& =\left(1-\left|\xi_{\sigma(\gamma)}^{\prime}\right|^{p}\right)+\left|\xi_{\sigma\left(\gamma_{1}\right)}^{\prime}+\frac{\xi_{\gamma_{1}}}{\left|\xi_{\gamma_{1}}\right|} \theta_{\gamma_{1}}\right|^{p} .
\end{aligned}
$$

于是，由 $V_{0}$ 等距的假设及引理 1 , 类似有

$$
\text { 上式 }=\left\|x+\frac{\xi_{\gamma_{1}}}{\left|\xi_{\gamma_{1}}\right|} e_{\gamma_{1}}\right\|^{p}=\left(1-\left|\xi_{\gamma_{1}}\right|^{p}\right)+\left|\xi_{\gamma_{1}}+\frac{\xi_{\gamma_{1}}}{\left|\xi_{\gamma_{1}}\right|}\right|^{p}=\left(1-\left|\xi_{\gamma_{1}}\right|^{p}\right)+\left(1+\left|\xi_{\gamma_{1}}\right|\right)^{p} \text {, }
$$

因而得到

$$
\left|\xi_{\sigma\left(\gamma_{1}\right)}^{\prime}+\frac{\xi_{\gamma_{1}}}{\left|\xi_{\gamma_{1}}\right|} \theta_{\gamma_{1}}\right|^{p}-\left|\xi_{\sigma\left(\gamma_{1}\right)}^{\prime}\right|^{p}=\left(1+\left|\xi_{\gamma_{1}}\right|\right)^{p}-\left|\xi_{\gamma_{1}}\right|^{p} .
$$

上式中, $\xi_{\sigma\left(\gamma_{1}\right)}^{\prime}$ 必与 $\frac{\xi_{\gamma_{1}}}{\left|\xi_{\gamma_{1}}\right|} \theta_{\gamma_{1}}$ 同号. 事实上, 反之则从上式导出 $\left|\xi_{\sigma\left(\gamma_{1}\right)}^{\prime}+\frac{\xi_{\gamma_{1}}}{\left|\xi_{\gamma_{1}}\right|} \theta_{\gamma_{1}}\right|^{p}-\left|\xi_{\sigma\left(\gamma_{1}\right)}^{\prime}\right|^{p}=\left(\left|\frac{\xi_{\gamma_{1}}}{\left|\xi_{\gamma_{1}}\right|} \theta_{\gamma_{1}}\right|-\left|\xi_{\sigma\left(\gamma_{1}\right)}^{\prime}\right|\right)^{p}-\left|\xi_{\sigma\left(\gamma_{1}\right)}^{\prime}\right|^{p}=\left(1-\left|\xi_{\sigma\left(\gamma_{1}\right)}^{\prime}\right|\right)^{p}-\left|\xi_{\sigma\left(\gamma_{1}\right)}^{\prime}\right|^{p} \leqslant 1$.

但注意到 $\xi_{\gamma_{1}} \neq 0$ 和 $p>1$, 故有

$$
\left(1+\left|\xi_{\gamma_{1}}\right|\right)^{p}-\left|\xi_{\gamma_{1}}\right|^{p}>\left(1+\left|\xi_{\gamma_{1}}\right|^{p}\right)-\left|\xi_{\gamma_{1}}\right|^{p}=1 .
$$

此显然与 (8) 式矛盾, 由此获证. 这样由 (8) 式导出: 对任意 $\xi_{\gamma_{1}} \neq 0$, 均有

$$
\left(1+\left|\xi_{\sigma\left(\gamma_{1}\right)}^{\prime}\right|\right)^{p}-\left|\xi_{\sigma\left(\gamma_{1}\right)}^{\prime}\right|^{p}=\left(1+\left|\xi_{\gamma_{1}}\right|\right)^{p}-\left|\xi_{\gamma_{1}}\right|^{p} .
$$

注意函数 $f(t)=(1+t)^{p}-t^{p}$ 是严格单调增的 $(p>1)$, 因此, 从 (3) 式立即得到 $\left|\xi_{\sigma\left(\gamma_{1}\right)}^{\prime}\right|=\left|\xi_{\gamma_{1}}\right|$. 最后, 令映像 $\pi=\sigma^{-1}$, 从上面结果导出: 对任意的 $\gamma \in \Gamma$, 存在惟一的 $\delta=\sigma(\gamma)$ 和绝对值为 1 的实数 $\theta_{\delta}$, 使得 $\xi_{\gamma}=\theta_{\delta} \xi_{\delta}^{\prime}$ (由保范性显然可知, 当 $\xi_{\gamma}=0$ 时, 必有 $\xi_{\delta}^{\prime}=\xi_{\sigma(\gamma)}^{\prime}=0$ ), 因此

$$
\xi_{\delta}^{\prime}=\frac{1}{\theta_{\delta}} \xi_{\gamma}=\theta_{\delta} \xi_{\pi(\delta)} .
$$

结合 (7) 和 (9) 式, 立即得到本定理结果.

有了上面关于 $V_{0}$ 的表现定理，我们不难导出有关的等距算子的延拓定理: 
定理 2 设 $V_{0}$ 为单位球面 $S_{1}\left[l^{p}(\Gamma)\right]$ 到 $S_{1}\left[l^{p}(\Delta)\right]$ 上的满等距算子 $(p>1)$, 那么, $V_{0}$ 必 可延拓为 $l^{p}(\Gamma)$ 到 $l^{p}(\Delta)$ 上的一个实线性等距算子.

证 当 $p=2$ 时, 结果已由作者 ${ }^{[2]}$ 证得. 下仅证 $p>1, p \neq 2$ 时的情形.

对任意 $x \in l^{p}(\Gamma)$, 令

$$
V(x)= \begin{cases}\|x\| V_{0}\left(\frac{x}{\|x\|}\right), & \text { 当 } x \neq \theta, \\ \theta, & \text { 当 } x=\theta .\end{cases}
$$

显然 $V$ 为算子 $V_{0}$ 的延拓. 下面证明 $V$ 亦为等距算子.

对任意两个非零元 $x_{1}, x_{2} \in l^{p}(\Gamma)$, 当设 $\frac{x_{1}}{\left\|x_{1}\right\|}=\sum_{\gamma \in \Gamma} \xi_{\gamma}^{(1)} e_{\gamma}$ 和 $\frac{x_{2}}{\left\|x_{2}\right\|}=\sum_{\gamma \in \Gamma} \xi_{\gamma}^{(2)} e_{\gamma}$ 时, 由 定理 1 可以得到

$$
V\left(\frac{x_{i}}{\left\|x_{i}\right\|}\right)=V_{0}\left(\frac{x_{i}}{\left\|x_{i}\right\|}\right)=\sum_{\delta \in \Delta} \theta_{\delta} \xi_{\pi(\delta)}^{(i)} d_{\delta}, \quad i=1,2,
$$

因此有

$$
\begin{aligned}
\left\|V\left(x_{1}\right)-V\left(x_{2}\right)\right\|^{p} & =\|\| x_{1}\left\|V_{0}\left(\frac{x_{1}}{\left\|x_{1}\right\|}\right)-\right\| x_{2}\left\|V_{0}\left(\frac{x_{2}}{\left\|x_{2}\right\|}\right)\right\|^{p} \\
& =\left\|x_{1}\right\|^{p} \cdot\left\|V_{0}\left(\frac{x_{1}}{\left\|x_{1}\right\|}\right)-\frac{\left\|x_{2}\right\|}{\left\|x_{1}\right\|} V_{0}\left(\frac{x_{2}}{\left\|x_{2}\right\|}\right)\right\|^{p} \\
& =\left\|x_{1}\right\|^{p} \cdot\left\|\sum_{\delta \in \Delta} \theta_{\delta} \xi_{\pi(\delta)}^{(1)} d_{\delta}-\frac{\left\|x_{2}\right\|}{\left\|x_{1}\right\|} \sum_{\delta \in \Delta} \theta_{\delta} \xi_{\pi(\delta)}^{(2)} d_{\delta}\right\|^{p} \\
& =\left\|x_{1}\right\|^{p} \cdot\left\|\sum_{\delta \in \Delta} \theta_{\delta}\left(\xi_{\pi(\delta)}^{(1)} d_{\delta}-\frac{\left\|x_{2}\right\|}{\left\|x_{1}\right\|} \xi_{\pi(\delta)}^{(2)}\right) d_{\delta}\right\|^{p} \\
& =\left\|x_{1}\right\|^{p} \cdot \sum_{\delta \in \Delta}\left|\theta_{\delta}\left(\xi_{\pi(\delta)}^{(1)} d_{\delta}-\frac{\left\|x_{2}\right\|}{\left\|x_{1}\right\|} \xi_{\pi(\delta)}^{(2)}\right)\right|^{p} \\
& =\left\|x_{1}\right\|^{p} \cdot \sum_{\delta \in \Delta}\left|\xi_{\pi(\delta)}^{(1)}-\frac{\left\|x_{2}\right\|}{\left\|x_{1}\right\|} \xi_{\pi(\delta)}^{(2)}\right|^{p} \\
& =\left\|x_{1}\right\|^{p} \cdot \sum_{\gamma \in \Gamma}\left|\xi_{\gamma}^{(1)}-\frac{\left\|x_{2}\right\|}{\left\|x_{1}\right\|} \xi_{\gamma}^{(2)}\right|^{p} \\
& =\left\|x_{1}\right\|^{p} \cdot\left\|\frac{x_{1}}{\left\|x_{1}\right\|}-\frac{\left\|x_{2}\right\|}{\left\|x_{1}\right\|} \cdot \frac{x_{2}}{\left\|x_{2}\right\|}\right\|^{p} \\
& =\left\|x_{1}-x_{2}\right\|^{p}, \quad \forall x_{1}, x_{2} \in l^{p}(\Gamma) .
\end{aligned}
$$

此即导出 $V$ 为空间 $l^{p}(\Gamma)$ 到 $l^{p}(\Delta)$ 上的一个满等距算子, 因此, 从 Mazur-Ulam 定理立即可知 $V$ 必为一个实线性的等距算子.

\section{参考文献}

1 Tingley D. Isometries of the unit sphere. Geometriae Dedicata, 1987, 22: 371 378

2 Ding G G. The 1-Lipschitz mapping between the unit spheres of two Hilbert spaces can be extended to a real linear isometry of the whole space. Science in China, Ser A, 2002, 45: 479 483

3 Mayer-Nieberg P. Banach Lattices. Berlin-Heildelberg-New York: Springer-Verlag, 1991

4 Banach S. Theoriě des opěrations Liněaires. Warszawa, Monografje Matematyczne, 1932 